\title{
Correlations between DNA methylation levels and nucleosome enrichment in the human genome
}

\author{
Clayton K Collings", John N Anderson \\ From Epigenetics and Chromatin: Interactions and processes \\ Boston, MA, USA. 11-13 March 2013
}

Next generation sequencing studies have contributed further evidence that the methylation of $\mathrm{CpG}$ dinucleotides can alter protein-DNA interactions, and this epigenetic mechanism is thought to play a critical role in the control of gene expression in higher eukaryotic organisms. Recent analyses of high resolution nucleosome maps have revealed novel relationships between DNA methylation and localization of nucleosomes $[1,2]$. In our latest work, we conducted high-throughput nucleosome reconstitution experiments on $572 \mathrm{~KB}$ of human DNA that was unmethylated or methylated by bacterial CpG methyltransferase in order to investigate the effects of this epigenetic modification on the positioning and stability of nucleosomes in vitro[2]. The results demonstrated that a subset of nucleosomes positioned by nucleotide sequence was sensitive to methylation where the modification increased the affinity of these sequences for the histone octamer. The features that distinguished these nucleosomes from the bulk of the methylation-insensitive nucleosomes were an increase in the frequency of $\mathrm{CpG}$ dinucleotides and a unique rotational orientation of $\mathrm{CpGs}$ such that their minor grooves tended to face toward the histones in the nucleosome rather than away. These methylation-sensitive nucleosomes were preferentially associated with exons as compared to introns while unmethylated $\mathrm{CpG}$ islands near transcription start sites became enriched in nucleosomes upon methylation. In order to provide additional validation for these findings, we examined the influence of $\mathrm{CpG}$ methylation levels [3] on the genome-wide distributions of nucleosomes reconstituted in vitro [4] derived from leukocyte DNA. Within DNA sequence contexts of high $\mathrm{G}+\mathrm{C}$ and $\mathrm{CpG}$ content, nucleosome occupancy was found not only to be enriched but also strongly correlated with levels of DNA methylation, and these optimal sequence environments were particularly accommodated by exons and CpG islands over other genomic components. Additionally, throughout the predominantly methylated genome, the minor grooves of methylated $\mathrm{CpG}$ dinucleotides favoured to lie inwards relative to the histone surface while unmethylated CpGs displayed no rotational orientation. The results in this report support our previous proposal, suggesting that DNA methylation is more directly involved in dictating nucleosome organization, especially in exons and $\mathrm{CpG}$ islands. Current efforts entailing comparisons of nucleosome assembly in cells to methylomes are underway to evaluate the effects of DNA methylation on the positioning and stability of nucleosomes in vivo.

\section{Published: 18 March 2013}

\section{References}

1. Chodavarapu $\mathrm{R}$, et al: Relationship between nucleosome positioning and DNA methylation. Nature 2010, 466:388-392.

2. Collings CK, Anderson JN: Effects of DNA methylation on nucleosome stability. Nucleic Acids Research 2013.

3. Li Y, et al: The DNA methylome of human peripheral blood mononuclear cells. PLoS Biology 2010, 8(11):e1000533.

4. Valouev $A$, et al: Determinants of nucleosome organization in primary human cells. Nature 2011, 474:516-520.

\section{doi:10.1186/1756-8935-6-S1-09}

Cite this article as: Collings and Anderson: Correlations between DNA methylation levels and nucleosome enrichment in the human genome. Epigenetics \& Chromatin 2013 6(Suppl 1):09. 